\title{
THE PRODUCT OF PRE-RADON MEASURES
}

\author{
Susumu OKaDa
}

\begin{abstract}
Let $\mu$ and $\nu$ be non- $\sigma$-Pinite pre-Radon measures on topological spaces $X$ and $Y$ respectively. Then there exists a unique pre-Radon measure $\lambda$ on the product space $X \times Y$ which satisfies $\lambda(A \times B)=\mu(A) \nu(B)$ for all Borel sets $A$ in $X$ and $B$ in $Y$ such that $\mu(A)<\infty$ and $\nu(B)<\infty$.
\end{abstract}

\section{Introduction}

Let $X$ be a topological space, $O(X)$ the family of open subsets of $X, F(X)$ the family of closed subsets, and $B(X)$ the Borel field, that is, the $\sigma$-algebra generated by $O(X)$. A Borel measure $\mu$ is said to be a pre-Radon measure if it satisfies the following conditions:

(i) for each $x \in X$, there is an open neighbourhood $U$ of $x$ such that $\mu(U)<\infty$;

(ii) for each $B \in B(X)$ with $\mu(B)<\infty$,

$$
\mu(B)=\sup \{\mu(E): B \supset F \in F(X)\} ;
$$

(iii) for each $B \in B(X)$,

$$
\mu(B)=\inf \{\mu(U): B \subset U \in O(X)\} ;
$$

(iv) for each increasing net $\left\{U_{\alpha}\right\} \subset O(X)$,

$$
\mu\left(U U_{\alpha}\right)=\sup _{\alpha} \mu\left(U_{\alpha}\right) \text {. }
$$

A Borel measure satisfying $(i)$ is called locally-bounded. If a Borel

Received 20 October 1981. 
measure satisfies (ii) (respectively (iii)), then we call it inner (respectively outer) regular. In particular, an inner and outer regular Borel measure is said to be regular.

A locally bounded measure $\mu$ is called a Radon measure if $\mu(B)=\sup \{\mu(K): K \subset B$ and $K$ is compact $\}$ for every $B \in B(X)$. Note that every Radón measure is semi-finite. Recall that a measure $\nu$ on a measurable space $(Y, B)$ is called semi-finite if

$$
\nu(A)=\sup \{\nu(B): A \supset B \in B, \nu(B)<\infty\}, A \in B \text {. }
$$

A measure space $(X, B, \mu)$ is called locally determined if $\mu$ is semi-finite and any subset $E$ of $X$ satisfying that $E \cap F \in B$ for all $F \in B$ with $\mu(F)<\infty$ belongs to $B$.

Suppose that $X$ is a topological space. A measure space $(X, A, \mu)$ is said to be a quasi-Radon measure space if it satisfies the following:

$$
\begin{aligned}
& \text { (i) }(X, A, \mu) \text { is complete and locally determined; } \\
& \text { (ii) } A \supset B(X) ; \\
& \text { (iii) if } E \in A \text { and } \mu(E)<\infty \text {, then there is a } G \in O(X) \\
& \text { such that } \mu(G)<\infty \text { and } \mu(E \cap G)>0 \text {; } \\
& \text { (iv) } \mu(E)=\sup \{\mu(E): E \supset F \in F(X)\} \text { for all } E \in A \text {; } \\
& \text { (v) for every increasing net }\left\{U_{\alpha}\right\} \subset O(X), \\
& \mu\left(U U_{\alpha}\right)=\sup \mu\left(U_{\alpha}\right) . \\
& \alpha
\end{aligned}
$$

As for its details, see Fremlin [3, \$72]. The relationship between preRadon measures and quasi-Radon measures is given by Amemiya, Okada and Okazaki [1, pp. 131-132].

From now on, all topological spaces are supposed to be Hausdorff.

In this paper, we study the product of two non- $\sigma$-finite pre-Radon measures.

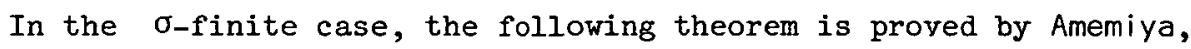
Okada and Okazaki $[1, \$ 9]$.

THEOREM 1.1. Let $\mu$ and $\nu$ be o-finite pre-Radon measures on topological spaces $X$ and $Y$ respectively. Then there exists a unique 
pre-Radon measure $\lambda$ on $X \times Y$ such that $\lambda(A \times B)=\mu(A) \nu(B)$ for every $A \in B(X)$ and every $B \in B(Y)$. Moreover, for a non-negative, extended real-valued Borel measurable function $f$ on $X \times Y$,

(i) $x \mapsto \int_{Y} f(x, y) d v(y)$ is $B(X)$-measurable,

(ii) $y \mapsto \int_{X} f(x, y) d \mu(x)$ is $B(Y)$-measurable,

(iii) $\int_{X} d \mu(x) \int_{Y} f(x, y) d \nu(y)=\int_{Y} d \nu(y) \int_{X} f(x, y) d \mu(x)$ $=\int_{X \times Y} f(x, y) d \lambda(x, y)$.

In the case of Radon measures, Bourbaki [2, $\$ 2$, no. 6] has shown the following theorem.

THEOREM 1.2. Let $\mu$ and $v$ be Radon measures on topological spaces $X$ and $Y$ respectively. Then there is a unique Radon measure $\lambda$ on $X \times Y$ such that $\lambda(A \times B)=\mu(A) \nu(B)$ for every $A \in B(X)$ and $B \in B(Y)$. Furthermore, for a non-negative, lower semi-continuous function $f$ on $X \times Y$,

(i) $x \mapsto \int_{Y} f(x, y) d \nu(y)$ and $y \mapsto \int_{X} f(x, y) d \mu(x)$ are zower semi-continuous on $X$ and $Y$ respectively, and

(ii) $\int_{X} d \mu(x) \int_{Y} f(x, y) d \nu(y)=\int_{Y} d \nu(y) \int_{X} f(x, y) d \nu(x)$ $=\int_{X \times Y} f(x, y) d \lambda(x, y)$.

Fremlin [4, Proposition 4.2] has shown the following theorem.

THEOREM 1.3. Let $(X, A, \mu)$ and $(Y, B, v)$ be two quasi-Radon measure spaces. Then there is a unique quasi-Radon measure $\lambda$ on $X \times Y$ such that $\lambda(A \times B)=\mu(A) \nu(B)$ for each $A \in A$ and $B \in B$.

In $\$ 2$, we shall show that the statement in Theorem 1.1 does not hold for non- $\sigma$-finite pre-Radon measures, in general; but we have a unique preRadon measure $\lambda$ on $X \times Y$ which satisfies the condition $\lambda(A \times B)=\mu(A) \cup(B)$ for all $A \in B(X)$ and $B \in B(Y)$ such that $\mu(A)<\infty$ and $\nu(B)<\infty$. 


\section{The product of pre-Radon measures}

Let $X$ be a set. A family $U$ of subsets of $X$ is said to be a paring if it satisfies the following conditions:

$$
\begin{aligned}
& \text { (i) } \emptyset \in U \text {; } \\
& \text { (ii) } U_{U \in U} U=x ; \\
& \text { (iii) if } U_{1}, U_{2} \in U \text {, then } U_{1} \cap U_{2}, U_{1} \cup U_{2} \in U .
\end{aligned}
$$

We denote by $R[u]$ the ring generated by. $u$.

The proof of the following lemma is straightforward.

LEMMA 2.1. Let $X$ be a set and $u$ a paving of subsets of $X$. Then, for a subset $E$ of $X, E \in \mathrm{R}[U]$ if and only if there are $v_{i}, w_{i} \in U(i=1,2, \ldots, n)$ such that

$$
\begin{aligned}
& \text { (i) } v_{i} \subset w_{i}(i=1,2, \ldots, n), \\
& \text { (ii) }\left(w_{i}-V_{i}\right) \cap\left(w_{j}-V_{j}\right)=\emptyset \text { if } i \neq j, \\
& \text { (iii) } E=\bigcup_{i=1}^{n}\left(w_{i}-V_{i}\right) .
\end{aligned}
$$

Let $(X, B, \mu)$ be a measure space. For $A \in B$, we can define a measure $\mu_{A}$ on $A \cap B$ as follows:

$$
\mu_{A}(A \cap B)=\mu(A \cap B), B \in B,
$$

where $A \cap B=\{A \cap B: B \in B\}$. We call $\mu_{A}$ the restriction of $\mu$ to $A$.

Let $(X, A, \mu)$ and $(y, B, \mu)$ be two totally finite measure spaces. Then we denote by $\mu \otimes \nu$ the product measure of $\mu$ and $\nu$. This $\mu \otimes \nu$ is defined on the product $\sigma$-algebra $A \otimes B$ and satisfies the condition $(\mu \otimes \nu)(A \times B)=\mu(A) \nu(B)$ for each $A \in A$ and each $B \in B$.

The following lemma is a fundamental tool.

LEMMA 2.2 (Amemiya, Okada and Okazaki [1, Theorem 3.1]). Let $X$ be a topological space and $u$ a paving generated by an open base of $X$. Let a non-negative, real-valued, finitely additive set function $m$ on 
$R[U]$ satisfy the following conditions:

(i) for every net $\left\{U_{\alpha}\right\} \subset U$ increasing to a $U \in U$,

$$
\lim _{\alpha} m\left(U_{\alpha}\right)=m(U) ;
$$

(ii) for each $U \in U$,

$$
m(U)=\sup \{m(F): U \supset F \in R[U] \cap F(X)\} .
$$

Then $m$ can be extended to a unique pre-Radon measure on $X$.

THEOREM 2.3. Let $\mu$ and $\nu$ be pre-Radon measures on topological spaces $X$ and $Y$ respectively. Then there is a unique pre-Radon measure $\lambda$ on $X \times Y$ satisfying the condition $\lambda(A \times B)=\mu(A) \nu(B)$ for alz $A \in B(X)$ and $B \in B(Y)$ such that $\mu(A)<\infty$ and $\nu(B)<\infty$.

Proof. Let $u$ be the paving generated by the open base $V=\{U \times V: U \in O(X), V \in O(X), \mu(U)<\infty, v(V)<\infty\}$. It follows from Lemma 2.1 that, for every $E \in \mathrm{R}[U]$, there is a $U \times V \in V$ such that $E \subset U \times V$. Hence we can define a set function $m$ on $\mathrm{R}[U]$ by

$$
m(E)=\left(\mu_{U} \otimes \nu_{V}\right)(E) \text {. }
$$

We claim that $m(E)$ is independent of the choice of $U \times V$. In fact, suppose that $E \subset U^{\prime} \times V^{\prime} \subset U \times V$ for another $U^{\prime} \times V^{\prime} \in V$, then it follows from the definition of product measures that

$\left(\mu_{U} \otimes v_{V}\right)_{U^{\prime} \times V^{\prime}}=\mu_{U^{\prime}} \otimes v_{V^{\prime}}$, which implies that $\left(\mu_{U^{\prime}} \otimes v_{V^{\prime}}\right)(E)=\left(\mu_{U} \otimes v_{V}\right)(E)$.

Given an increasing net $\left\{W_{\alpha}\right\} \subset U$ such that $\bigcup_{\alpha} W_{\alpha}=W \in U$, there exists a set $U \times V \in V$ such that $W \subset U \times V$. It follows from Theorem 1.1 that $m(W)=\left(\mu_{U} \otimes v_{V}\right)(W)=\sup _{\alpha}\left(\mu_{U} \otimes v_{V}\right)\left(W_{\alpha}\right)=\sup _{\alpha} m\left(W_{\alpha}\right)$.

Given $W=\bigcup_{i=1}^{n}\left(U_{i} \times v_{i}\right) \in u$ with $U_{i} \times v_{i} \in v$, and $\varepsilon>0$, there are $E_{i} \in F(X)$ with $F_{i} \subset U_{i}, G_{i} \in F(X)$ with $G_{i} \subset V_{i}$ such that $\mu\left(U_{i}-F_{i}\right)<\varepsilon / n\left(\nu\left(V_{i}\right)+1\right)$ and $\nu\left(V_{i}-G_{i}\right)<\varepsilon / n\left(\mu\left(U_{i}\right)+1\right)$ for all $i=1,2, \ldots, n$ since both $\mu$ and $\nu$ are regular. Let 


$$
\begin{aligned}
F=\bigcup_{i=1}^{n}\left(F_{i} \times G_{i}\right) & \in F(X \times Y) ; \text { then } \\
m(W-F) & \left.\leq m \int_{i=1}^{n}\left(U_{i} \times V_{i}-F_{i} \times G_{i}\right)\right) \\
& \leq \sum_{i=1}^{n}\left(m\left(U_{i} \times V_{i}\right)-m\left(E_{i} \times G_{i}\right)\right) \\
& \leq \sum_{i=1}^{n} 2 \varepsilon / n=2 \varepsilon .
\end{aligned}
$$

Then it follows from Lemma 2.2 that there is a unique pre-Radon measure $\lambda$ on $X \times Y$ such that $\lambda=m$ on $\mathrm{R}[U]$. For each $A \in B(X)$ with $\mu(A)<\infty$, and each $B \in B(Y)$ with $\nu(B)<\infty$, there exists a $U \times V \in V$ such that $U \times V \supset A \times B$. Then $\lambda_{U \times V}$ is a pre-Radon measure on $U \times V$ by Amemiya, Okada and Okazaki [1, Theorem 5.2]. If we denote by $\overline{\mu_{U} \otimes \nu_{V}}$ the pre-Radon extension of $\mu_{U} \otimes \nu_{V}$ on $U \times V$, then $\lambda_{U \times V}$ coincides with $\overline{\mu_{U} \otimes v_{V}}$ on $\mathrm{R}\left[u_{U \times V}\right]$, where $u_{U \times V}$ is the paving generated by $\left\{U_{1} \times V_{1}: U_{1} \in O(U), V_{1} \in O(V)\right\}$. By Lemma 2.2, $\lambda_{U \times V}=\overline{\mu_{U} \otimes V_{V}}$ on $U \times V$, so that

$$
\begin{aligned}
\lambda(A \times B) & =\lambda_{U \times V}(A \times B) \\
& =\overline{\mu_{U} \otimes v_{V}}(A \times B) \\
& =\mu_{U}(A) \nu_{V}(B) \\
& =\mu(A) \nu(B) .
\end{aligned}
$$

The uniqueness of $\lambda$ is obvious. This completes the proof.

REMARK 2.4. In the above theorem, if both $\mu$ and $\nu$ are semifinite, then $\lambda(A \times B)=\mu(A) \nu(B)$ even when $A \in B(X)$ with $\mu(A)=\infty$ and $B \in B(Y)$ with $\nu(B)=\infty$. In fact, given a natural number $N$, there are an $A_{0} \in B(X)$ with $A_{0} \subset A$ and a $B_{0} \in B(Y)$ with $B_{0} \subset B$ such that $\mu\left(A_{0}\right)>N$ and $\mu\left(B_{0}\right)>N$. Then

$$
\lambda(A \times B) \geq \lambda\left(A_{0} \times B_{0}\right)=\mu\left(A_{0}\right) \nu\left(B_{0}\right)>N^{2},
$$

which implies $\lambda(A \times B)=\mu(A) \nu(B)=\infty$. 
The following example shows that we cannot have $\lambda(A \times B)=\mu(A) \nu(B)$ for some $A \in B(X)$ with $\mu(A)=0$ and $B \in B(Y)$ with $\mu(B)=\infty$.

EXAMPLE 2.5. Let $X=[0,1]$ with the usual topology and $y=[0,1]$ with the discrete topology. Suppose that $C \subset Y$ is not Lebesgue measurable. We define a measure $V$ on $Y$ by

$$
\nu(B)=\text { the cardinality of } B \cap C
$$

for $B \subset Y$. Then $\nu$ is a Radon measure as well as a semi-finite preRadon measure. Let $\mu$ be the Lebesgue measure on $X$. By Theorem 2.3, there is a unique pre-Radon measure $\lambda$ on $X \times Y$ satisfying the condition $\lambda(A \times B)=\mu(A) \nu(B)$ for all $A \in B(X)$ and $B \in B(Y)$ such that $\mu(A)<\infty$ and $\nu(B)<\infty$. Fix an $x_{0} \in X$. Then $\mu\left(\left\{x_{0}\right\}\right) \nu(Y)=0 . \infty=0$. On the other hand, take any open subset $W$ of $X \times Y$ which includes the set $\left\{x_{0}\right\} \times Y$. Let

$$
Y_{n}=\{y \in Y: \mu(W(y)) \geq 1 / n\}, \quad n=1,2, \ldots,
$$

where $W(y)=\{x \in X:(x, y) \in W\}$. Since $Y$ is equal to the union of $\left\{Y_{n}: n=1,2, \ldots\right\}$, there exists a natural number $n$ for which $Y_{n} \cap C$ is an infinite set. Hence $\lambda(W)=\infty$ since $W$ includes the union of the family $\left\{W(y) \times\{y\}: y \in Y_{n} \cap C\right\}$. Since $\lambda$ is outer regular, we have $\lambda\left(\left\{x_{0}\right\} \times Y\right)=\infty$; in other words, $\lambda\left(\left\{x_{0}\right\} \times Y\right) \neq \mu\left(\left\{x_{0}\right\}\right) \nu(Y)$.

Furthermore, this example shows that the statement in Theorem 1.1 does not always hold for the non- $\sigma$-finite case. In fact, let $f$ be the characteristic function of the Borel subset $E=\{(x, x): x \in[0,1]\}$ of $X \times Y$. Then the function $x \mapsto \int_{Y} f(x, y) d \nu(y)$ is not u-measurable.

REMARK 2.6. In the above example, there is a Radon measure $\rho$ on $X \times Y$ such that $\rho(A \times B)=\mu(A) \nu(B)$ for each $A \in B(X)$ and $B \in B(Y)$ by Theorem 1.2. Further, note that $\left(X \times Y, \overline{B(X \times Y)}, \rho^{*}\right)$ is a quasiRadon measure space, where $\rho^{*}$ is the outer measure derived from $\rho$, $\overline{B(\overline{X \times Y})}$ is the completion of $B(X \times Y)$ with respect to $\rho$. For every compact set $K$, there exists a finite subset $\left\{y_{1}, y_{2}, \ldots, y_{n}\right\}$ of $Y$ such that $K=\bigcup_{i=1}^{n} K\left(y_{i}\right) \times\left\{y_{i}\right\}$. So $\lambda(K)=\sum_{i=1}^{n} \mu\left(K\left(y_{i}\right)\right) v\left(\left\{y_{i}\right\}\right)$. Thus, 
given a Borel subset $B$ of $X \times Y$, we have

$$
\begin{aligned}
\rho(B) & =\sup \{\rho(K): K \leftarrow B \text { and } K \text { is compact }\} \\
& =\sup \{\lambda(K): K \subset B \text { and } K \text { is compact }\} \leq \lambda(B) .
\end{aligned}
$$

We claim that $\rho$ is different from $\lambda$. Indeed, $\rho(E)=0$ while $\lambda(E)>0$ for the diagonal set $E$.

\section{References}

[1] Ichiro Amemiya, Susumu Okada and Yoshiaki Okazaki, "Pre-Radon measures on topological spaces", Kodai Math. J. 1 (1978), 101-132.

[2] N. Bourbaki, Éléments de mathématique. Fasc. XXXV. Livre VI: Intégration. Chapitre IX: Intégration sur les espaces topologiques séparés (Actualités Scientifiques et Industrielles, 1343. Hermann, Paris, 1969).

[3] D.H. Fremlin, Topological Riesz spaces and measure theory (Cambridge University Press, Cambridge, 1974).

[4] D.H. Fremlin, "Quasi-Radon measure spaces", unpublished manuscript.

School of Mathematical Sciences,

Flinders University of South Australia,

Bedford Park,

South Australia 5042,

Australia. 